\title{
Cimex lectularius Linnaeus, 1758 (Hemiptera: Cimicidae) in Costa Rica: First Case Report Confirmed by Molecular Methods in Central America
}

\author{
Juan Carlos Cambronero-Heinrichs, ${ }^{1,3}$ Laura Sofía Sánchez-Portilla, ${ }^{2}$ \\ Ólger Calderón-Arguedas, ${ }^{1}$ and Adriana Troyo'
} 'Centro de Investigación en Enfermedades Tropicales (CIET), Facultad de Microbiología, Universidad de Costa Rica, Sede Rodrigo
Facio Brenes, Montes de Oca, San José, Costa Rica, 11501-2060, ${ }^{2}$ Escuela de Biología, Universidad de Costa Rica, Sede Rodrigo Facio
Brenes, Montes de Oca, San José, Costa Rica, 11501-2060, and ${ }^{3}$ Corresponding author, e-mail: juan.cambroneroheinrichs@ucr.ac.cr

Subject Editor: Roberto Pereira

Received 11 September 2019; Editorial decision 2 December 2019

\begin{abstract}
Cimex lectularius and Cimex hemipterus are the most common species of bedbugs that infest homes. Although case reports decreased substantially by the end of the 20th century, bed bugs, and especially $C$. lectularius, are currently suffering a resurgence mostly attributed to insecticide resistance, inadequate pest control, and increased travel. Here, we report, to the best of our knowledge, the first molecular confirmation of $C$. lectularius in Central America. Specimens were obtained from an apartment located in Heredia, Costa Rica. These specimens were identified morphologically as $C$. lectularius. The species identification was confirmed by amplifying and sequencing fragments of the cytochrome oxidase subunit I (COI) and the 16S rRNA (16S) genes. The phylogenetic analysis showed that the sequences obtained were more closely related to a $C$. lectularius mitochondrial complete genome sequence from China, with similarities of $98.84 \%$ (686/694) for COI and $98.97 \%$ $(387 / 391)$ for $16 \mathrm{~S}$. The finding of $C$. lectularius in Costa Rica will require further investigation in order to determine the extent of current infestations and the susceptibility to insecticides, especially due to the impact that this species can have in human health, as well as the tourism industry in the region.
\end{abstract}

Key words: Cimicidae, Cimex lectularius, bedbugs, Central America, Costa Rica

Cimicidae is a family of cosmopolitan insects that are temporary but obligate hematophagous ectoparasites and feed at night from hosts that mainly include humans, bats, and birds (Reinhardt and Siva-Jothy 2007, Zorrilla-Vaca et al. 2015) The species adapted to feeding on human blood, known as bedbugs, are Cimex lectularius, which is found mainly in temperate latitudes, and Cimex hemipterus Fabricius, 1803 (Hemiptera: Cimicidae) which is prevalent in tropical and subtropical regions (Koganemaru and Miller 2013). This parasitic relationship has been registered even in millennia-old Egyptian archeological sites (Panagiotakopulu and Buckland et al. 1999), becoming widespread and common all over the world in the first half of the 20th century (Potter 2011). With the discovery and repeated use of insecticides such as DDT (dichloro-diphenyl trichloroethane), bed bug infestations declined dramatically by the end of the 20th century (Doggett et al. 2012). Unfortunately, bedbugs, and especially C. lectularius, have resurged notably over the past decade in many areas of the world, mostly due to an increase in insecticide resistance, inadequate pest control, and international travel (Dang et al. 2017).
For example, both bedbugs are highly resistant to pyrethroids worldwide, and recent studies have revealed that pyrethroid-resistance-associated mutations $(\mathrm{kdr})$ are frequent in C. lectularius populations from Europe and the United States (Dang et al. 2015, Balvin and Booth 2018, Holleman et al. 2019), and this evidence should influence control strategies applied to new infestations.

In the past decade, C. lectularius infestations in the Americas were registered in many countries, including Canada, United States, Venezuela, Peru, Argentina, and Chile (Zorrilla-Vaca et al. 2015). During that same period of time, there were no published records of bed bug occurrence in Central America, with the exception of a single report from Nicaragua, where no species identification was conducted (Bendaña-García 2018). Here, we present the first report of a C. lectularius infestation confirmed by molecular methods in a property in Costa Rica and Central America, which represents a case of bed bug resurgence in an area where C. hemipterus was the prevalent species. This finding may represent the initial stages of invasion, although it is also possible that other infestations are 
being misidentified as C. hemipterus, given its predominantly tropical distribution.

\section{Methods}

Insect Sampling and Morphological Identification

Bedbugs were obtained from crevices of a bed at an apartment in Heredia, Costa Rica, in June 2017. There, the inhabitants reported a rash in feet and hands and insomnia, as a result of the insect bites. Live insects were flushed out by heating the piece of wooden furniture with a hairdryer. Specimens that came out were collected and stored in $70 \%$ ethanol at $-20^{\circ} \mathrm{C}$, prior to identification. Insects were identified morphologically by direct observation of key characters (Fig. 1) under a dissecting stereo microscope (CDC 1967, Campbell et al. 2016).

\section{DNA Extraction and Molecular Analyses}

One nymph was macerated in a microcentrifuge tube using a sterile pestle and resuspended in $1 \mathrm{ml}$ of sterile saline solution $(0.9 \% \mathrm{NaCl})$. Genomic DNA extraction was carried out using the NucleoSpin Tissue Kit (Macherey-Nagel), according to the manufacturer's instructions. For molecular identification, one-step PCR protocols were employed to amplify fragments of two mitochondrial genes: the cytochrome oxidase subunit I (COI) gene, using primers LepF (5'-ATT CAA CCA ATC ATA AAG ATA TNG G-3') and LepR (5'-TAW ACT TCW GGR TGT CCR AAR AAT CA-3'; 712 bp product); and the 16S rRNA gene (16S), using primers LR-J-13007 (5'-TTA CGC TGT TAT CCC TAA-3') and LR-N-13398 (5'-CGC CTG TTT ATC AAA AAC AT-3'; 391 bp product; Balvín et al. 2012). PCR products were purified using ExoSAP-IT (Applied Biosystems), and they were sent to Macrogen Inc. (Seoul, South Korea) for sequencing. DNA sequences
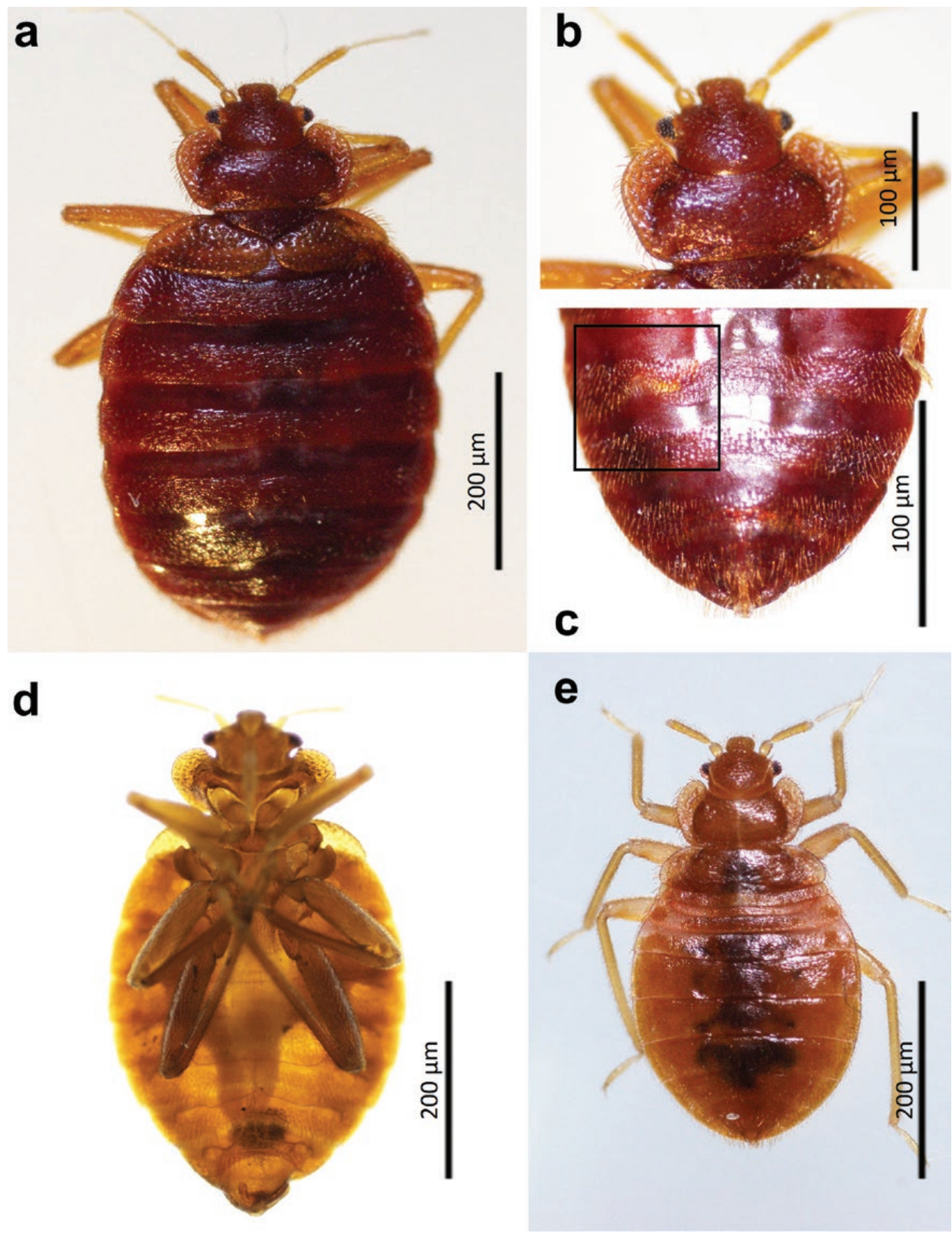

Fig. 1. Cimex lectularius specimens collected in Heredia, Costa Rica. (a) Dorsal view of a female adult, (b) head and pronotum with a deeply excavated anterior margin, (c) ventral view of female abdomen showing the paragenital sinus, (d) ventral of a male adult, and (e) dorsal view of a nymph. 
were edited and assembled with DNA Baser (Heracle BioSoft), and they were identified by sequence homology to publically available sequences using the Basic Local Alignment Search Tool (BLAST). Sequences obtained were deposited in GenBank with Accession numbers MN265385 (16S) and MN271345 (COI).

A concatenated phylogenetic tree of both $16 \mathrm{~S}$ and COI genes (Fig. 2) and two individual trees (Supp Material [online only]) for both genes were constructed as described by Cambronero-Heinrichs et al. (2019). Briefly, existing Cimex spp. sequences were retrieved from GenBank, sequence alignment was done separately for every gene on the MAFFT 7 CRBC Server, the aligned sequences were adjusted to the same length in Mega 7, and they were concatenated manually. The phylogenetic analysis was performed employing the Bayesian method with Mr. Bayes software, using 2 million generations and setting the burn-in fraction to 0.25 . The Bayesian information criterion was selected, and final editions of the tree were made using Mega7.

\section{Results and Discussion}

In total, 25 bed bug specimens, 16 nymphs and 9 adults ( 4 males and 5 females), were collected and identified preliminarily as C. lectularius, based on the presence of a pronotum with a deeply excavated anterior margin (Fig. 1; CDC 1967, Campbell et al. 2016). This morphological identification was confirmed by sequencing of partial fragments of the COI and $16 \mathrm{~S}$ genes from one specimen. The partial sequences of COI (712 bp) and 16S (391 bp) obtained showed high similarity on BLAST to C. lectularius sequences from Europe and China (Table 1). Moreover, the phylogenetic analysis with concatenated sequences indicated that specimens collected in Costa Rica were more closely related to C. lectularius from China (Fig. 2). However, it is important to note that among the sequences of C. lectularius in GenBank, none of them are from countries of Latin America. Furthermore, we want to highlight that to avoid mis-identification morphological and barcoding identification should be conducted together.

Results show that C. lectularius is present in Costa Rica. Although this species has a cosmopolitan distribution, it has been found mainly in temperate regions of the world (Zorrilla-Vaca et al. 2015), whereas the tropical bed bug, C. hemipterus, was the one previously reported throughout tropical and subtropical regions, including Central America (Usinger 1966). Moreover, this infestation outside of the assumed range of this species is comparable to reports of C. hemipterus on continental North America, outside what is considered its natural range (Campbell et al. 2016).

Historical evidence from Central America confirms that C. hemipterus was the species prevalent in the region, including Nicaragua, Costa Rica, and Panama (Usinger 1966). Moreover, previous to this report, the Collection of Medical Entomology at the University of Costa Rica had only C. hemipterus specimens from different areas of the country, and most were obtained before the $1980 \mathrm{~s}$ (the most recent specimen was collected in 1998; Adriana Troyo,

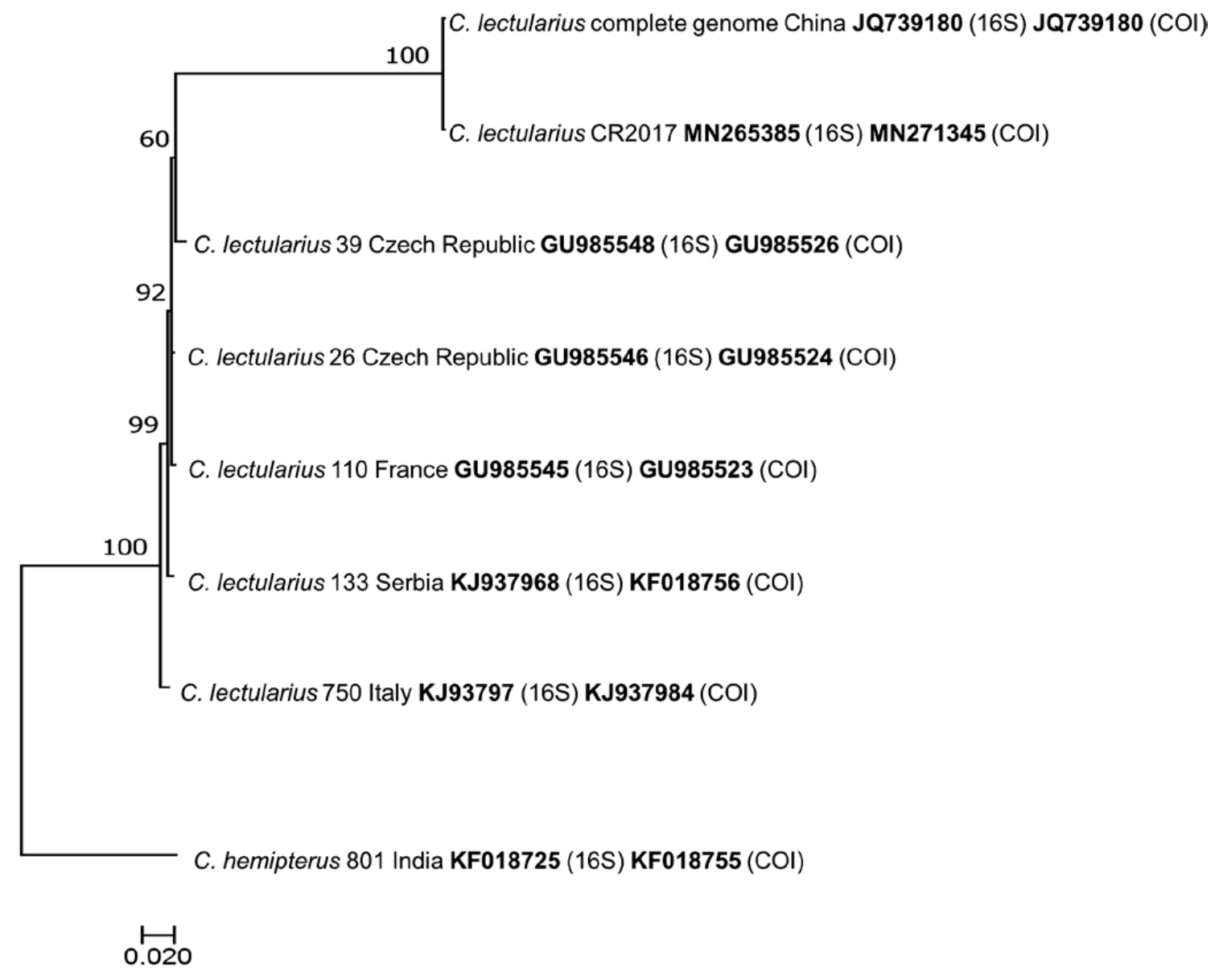

Fig. 2. Phylogenetic analysis based on the concatenated COI and $16 \mathrm{~S}$ gene fragments of selected Cimex spp. sequences (GenBank) and $C$. lectularius from Costa Rica (this study). Scale bars represent number of substitutions per nucleotide position. The numbers above the branches represent their Bayesian-calculated posterior probabilities. For individual trees for both genes see Supp Figs. S1 and S2 (online only). 
Table 1. Similarity of Cimex lectularius from Costa Rica (CR2017) with close relative sequences from GenBank

\begin{tabular}{llrr}
\hline ID & Country & $\begin{array}{c}16 \text { S } \\
\text { \% similarity (bp) }\end{array}$ & $\begin{array}{c}\text { COI } \\
\% \text { similarity (bp) }\end{array}$ \\
\hline Complete Genome & & $98.97(387 / 391)$ & $98.84(686 / 694)$ \\
26 & China & $99.47(377 / 379)$ & $99.09(652 / 658)$ \\
39 & Czech Republic & $99.47(377 / 379)$ & $99.09(652 / 658)$ \\
110 & Czech Republic & $99.21(376 / 379)$ & $99.24(653 / 658)$ \\
133 & France & $99.47(378 / 380)$ & $98.78(649 / 657)$ \\
750 & Serbia & $99.21(377 / 380)$ & $98.18(646 / 658)$ \\
\hline
\end{tabular}

personal communication). There have been no confirmed reports of bed bug infestations in Costa Rica for more than $20 \mathrm{yr}$, until now.

The global resurgence of Cimex spp. was reviewed by ZorrillaVaca et al. (2015), and they show that recent reports from all over the world follow a similar pattern as to the historical distribution of both species, where C. lectularius is documented mainly in nearctic and palearctic areas, with the exception of a few cases from Papua New Guinea and tropical countries from Africa and Asia. To the best of our knowledge, this is the first description of a C. lectularius infestation, confirmed by molecular methods, in Central America.

In the case of Costa Rica, it is possible that the recent introduction of C. lectularius is related to tourism or immigration. Given that adult and nymphs of bedbugs or their eggs can be easily transported in packed clothes, furniture, and other fomites, the ectoparasite resurgence around the world has been associated with the increase in international travel due to globalization (Doggett et al. 2012, Zorrilla-Vaca et al. 2015). However, it is difficult to track the cause or origin of the specific infestation discussed here, mainly because there is no mandatory surveillance concerning Cimex in Costa Rica, or other countries of Central America. In addition, it is not possible to compare barcoding information from neighboring countries, since there are no other sequences of C. lectularius from Latin America available on GenBank.

With the possible resurgence of Cimex spp. in Central America, there is growing concern as to the impact of infestations in the local economy and human health. Tourism, for example, is responsible for the largest foreign exchange income in Costa Rica, even more than agriculture and exports (Inman and Prado 2002). Bed bug infestations result not only in a bad reputation of affected hotels, but also mean significant costs in terms of control methods (Koganemaru and Miller 2013). Furthermore, the repetitive bite of the insect can cause skin reactions (itch, wheal, and lesions), allergic responses that may trigger anaphylaxis, secondary infections, discomfort, and psychological distress, such as insomnia and anxiety (Rahim et al. 2016). Moreover, although most evidence is inconclusive or does not support a role for bedbugs as pathogen vectors, there is always concern about their potential involvement in disease transmission (Goddard and DeShazo 2009).

This case report represents a warning to the entomological surveillance systems in Costa Rica and the rest of Central America, about the presence of C. lectularius in the region. It is clear that more research about this topic is needed, in order to prevent and control infestations by cimicids.

\section{Supplementary Data}

Supplementary data are available at Journal of Medical Entomology online.

\section{Acknowledgments}

We thank Rolando Moreira for preliminary laboratory analyses and support, as well as Ivan Coronado for technical assistance and José Pedro Sánchez for all the help during sampling. We thank Brittany Campbell for providing helpful information and guidance for morphological identification of species. This research was funded in part by Universidad de Costa Rica projects B5735 and ED548. The authors declare no conflict of interest.

\section{References Cited}

Balvín, O., and W. Booth. 2018. Distribution and frequency of pyrethroid resistance-associated mutations in host lineages of the bed bug (Hemiptera: Cimicidae) across Europe. J. Med. Entomol. 55: 923-928.

Balvín, O., P. Munclinger, L. Kratochvíl, and J. Vilímová. 2012. Mitochondrial DNA and morphology show independent evolutionary histories of bedbug Cimex lectularius (Heteroptera: Cimicidae) on bats and humans. Parasitol. Res. 111: 457-469.

Bendaña-García, G. 2018. Alarmante brote de jelepates en managua y departamentos. Temas Nicaragüenses. 120: 408-417.

Cambronero-Heinrichs, J. C., B. Matarrita-Carranza, C. Murillo-Cruz, E. Araya-Valverde, M. Chavarría, and A. A. Pinto-Tomás. 2019. Phylogenetic analyses of antibiotic-producing Streptomyces sp. isolates obtained from the stingless-bee Tetragonisca angustula (Apidae: Meliponini). Microbiology. 165: 292-301.

Campbell, B. E., Koehler, P. G., Buss, L. J., and Baldwin, R. W. 2016. Recent documentation of the tropical bed bug (Hemiptera: Cimicidae) in Florida since the common bed bug resurgence. Florida Entomologist. 99: 549-552.

Center for Disease Control and Prevention (CDC). 1967. Pictorial keys to arthropods, reptiles, birds, and mammals of public health significance title. US Department of Health \& Human Services-CDC, USA.

Dang, K., C. S. Toi, D. G. Lilly, C. Y. Lee, R. Naylor, A. Tawatsin, U. Thavara, W. Bu, and S. L. Doggett. 2015. Identification of putative kdr mutations in the tropical bed bug, Cimex hemipterus (Hemiptera: Cimicidae). Pest Manag. Sci. 71: 1015-1020.

Dang, K., S. L. Doggett, G. Veera Singham, and C. Y. Lee. 2017. Insecticide resistance and resistance mechanisms in bed bugs, Cimex spp. (Hemiptera: Cimicidae). Parasites and Vectors. 10: 1-31.

Doggett, S. L., D. E. Dwyer, P. F. Peñas, and R. C. Russell. 2012. Bed bugs: clinical relevance and control options. Clin. Microbiol. Rev. 25: 164-192.

Donnelly, M. J., V. Corbel, D. Weetman, C. S. Wilding, M. S. Williamson, and W. C. Black, $4^{\text {th }} .2009$. Does $\mathrm{kdr}$ genotype predict insecticide-resistance phenotype in mosquitoes? Trends Parasitol. 25: 213-219.

Goddard, J., and R. deShazo. 2009. Bed bugs (Cimex lectularius) and clinical consequences of their bites. JAMA. 301: 1358-1366.

Holleman, J. G., Robison, G. A., Bellovich, I. J., and Booth, W. 2019. Knockdown resistance-associated mutations dominate populations of the common bed bug (Hemiptera: Cimicidae) across the South Central United States. J. Med. Entomol. 56: 1678-1683.

Inman, C., K. Flores, and A. Prado. 2002. Tourism in Costa Rica. INCAE, Costa Rica.

Koganemaru, R., and D. M. Miller. 2013. The bed bug problem: past, present, and future control methods. Pestic. Biochem. Physiol. 106: 177-189.

Panagiotakopulu, E., and P. C. Buckland. 1999. Cimex lectularius L., the common bed bug from pharaonic Egypt. Antiquity. 73: 908-911.

Potter, M. F. 2011. The history of bed bug management. Am. Entomol. 57: $14-25$. 
Rahim, A. H. A., Z. Zahran, and A. H. A. Majid. 2016. Human skin reactions towards bites of tropical bed bug, Cimex hemipterus F. (Hemiptera: Cimicidae): a preliminary case study. Asian Pacific J. Trop. Dis. 6: 366-371. Reinhardt, K., and M. T. Siva-Jothy. 2007. Biology of the bed bugs (Cimicidae). Annu. Rev. Entomol. 52: 351-374.
Usinger, R. L. 1966. Monograph of Cimicidae. Entomological Society of America, Washington DC.

Zorrilla-Vaca, A., M. M. Silva-Medina, and K. Escandón-Vargas. 2015. Bedbugs, Cimex spp.: their current world resurgence and healthcare impact. Asian Pacific J. Trop. Dis. 5: 342-352. 